\title{
HIGHLIGHTS
}

MINERAL METABOLISM

\section{Sevelamer slows calcification and reduces levels of AGEs}

A randomized trial has shown that sevelamer decreases the risk of calcification and reduces the accumulation of advanced glycation end products (AGEs) in patients undergoing maintenance hemodialysis.

High serum levels of phosphorus, calcium, and calcium-phosphorus product are associated with increased risk of arterial calcification and cardiovascular mortality. In addition, high levels of circulating AGEs are common in uremic patients and have been related to cardiovascular disease. Sevelamer is a mineral-free phosphate binder that has been shown to decrease serum levels of phosphorus and parathyroid hormone in dialysis patients, and to attenuate the progression of calcification. Takatoshi Kakuta et al. compared the effects of sevelamer and calcium carbonate on the progression of arterial calcification and on plasma concentrations of the AGE pentosidine.

Of 200 screened hemodialysis patients with a mean dialysis vintage of $118 \pm 89$ months, 183 were randomly assigned to open-label treatment with sevelamer $(n=91)$ or calcium carbonate $(n=92)$ for 12 months; 163 patients were included in the final analysis. Serum levels of calcium, phosphorus, parathyroid hormone, and lipids were measured at baseline and then every 2 weeks during the study. Plasma pentosidine concentration was determined at study entry, 6 months after randomization, and at the end of the study. Changes in calcification were evaluated using multislice CT and coronary artery calcification score (CACS).

Absolute values of CACS increased in both groups, but the mean change in CACS was significantly smaller in the sevelamer group than in the calcium carbonate group after adjusting for baseline values. The proportion of participants with a $\geq 15 \%$ increase in CACS was also smaller in the sevelamer group than in the calcium carbonate group (35\% versus 59\%, respectively). Most biochemical parameters showed similar trends in both groups, although levels of LDL cholesterol decreased significantly in the sevelamer group, but not in the calcium carbonate group. Plasma pentosidine levels increased significantly from baseline in the calcium

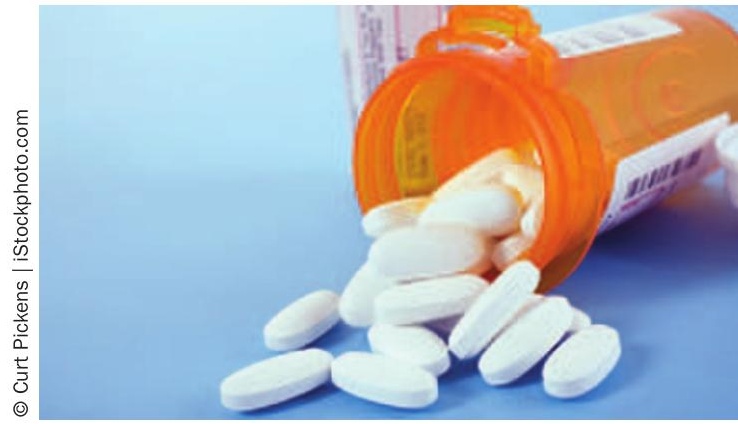

carbonate group, and at study completion were significantly higher than levels in the sevelamer group.

Results from this trial show that sevelamer reduces the risk of calcification. "Our findings indicate that sevelamer has other potential benefits, and the possibility is that it removes AGEs or suppresses their production," says Kakuta. "We are interested in exploring whether a reduction in AGEs slows coronary artery calcification. If it does, medications that interfere with the production of AGEs could be used in patient care to reduce the risk of coronary artery calcification."

Helene Myrvang

Original article Kakuta, T. et al. Effect of sevelamer and calcium-based phosphate binders on coronary artery calcification and accumulation of circulating advanced glycation end products in hemodialysis patients. Am. J. Kidney Dis. 57, 422-431 (2011) 